\title{
Differential expression of up-regulated cofilin-1 and down-regulated cofilin-2 characteristic of pancreatic cancer tissues
}

\author{
YUFENG WANG $^{1}$, YASUHIRO KURAMITSU ${ }^{1}$, TOMIO UENO ${ }^{2}$, NOBUAKI SUZUKI ${ }^{2}$, SHIGEFUMI YOSHINO ${ }^{2}$, \\ NORIO IIZUKA $^{2}$, XIULIAN ZHANG ${ }^{1}$, MASAAKI OKA ${ }^{2}$ and KAZUYUKI NAKAMURA ${ }^{1}$ \\ Departments of ${ }^{1}$ Biochemistry and Functional Proteomics and ${ }^{2}$ Digestive Surgery of Applied Molecular Bioscience, \\ Yamaguchi University Graduate School of Medicine, Ube, Yamaguchi 755-8505, Japan
}

Received June 29, 2011; Accepted July 28, 2011

DOI: $10.3892 /$ or.2011.1447

\begin{abstract}
Pancreatic cancer (PC) is one of the most deadly malignant tumors. The aim of this study was to identify potential biomarkers for PC. Using two-dimensional gel electrophoresis and liquid chromatography-tandem mass spectrometry, the proteomic profiles of pancreatic cancerous and non-cancerous tissues from ten patients with PC were compared. One of the numerous spots that showed stronger intensity in cancerous compared to non-cancerous tissues was identified as non-muscle cofilin (cofilin-1). This up-regulation was validated by Western blot analysis. It is noteworthy that Western blot analysis showed significantly lower expression of muscle cofilin (cofilin-2) in pancreatic cancerous tissues compared to non-cancerous tissues. This is the first time that cofilin isoforms (cofilin-1/2) have been identified to be differentially expressed in pancreatic cancerous tissues. Therefore, cofilin isoforms may serve as candidates for clinically useful biomarkers or therapeutic targets for PC.
\end{abstract}

\section{Introduction}

Pancreatic cancer (PC) is a malignant tumor which is associated with an extremely unfavorable prognosis. Because of delayed diagnosis and the lack of response to various therapies, few patients with PC survive for more than 5 years; due to rapid aggressiveness, most cases are diagnosed after metastatic spread (1). Therefore, it is critical to discover more sensitive biomarkers for the diagnosis of patients with $\mathrm{PC}$, and the biological mechanisms involved in the extreme aggressiveness of PC should be clarified.

Correspondence to: Dr Yasuhiro Kuramitsu, Department of Biochemistry and Functional Proteomics, Yamaguchi University Graduate School of Medicine, 1-1-1 Minami-Kogushi, Ube, Yamaguchi 755-8505, Japan

E-mail: climates@yamaguchi-u.ac.jp

Key words: two-dimensional gel electrophoresis, liquid chromatography-tandem mass spectrometry, pancreatic cancer, cofilin-1/2
Proteomics has been widely applied to discover candidate biomarkers in various types of cancers. Using proteomic differential display analysis, research groups have identified biomarkers such as PEA-15 in human malignant pleural mesothelioma cell lines, heat-shock proteins (Hsp27, Hsp70, GRP78) in hepatocellular carcinoma, numerous candidate proteins in colorectal cancer and $\alpha$-1-antitrypsin isoforms as a possible serum biomarker in pancreatic cancer (2-6). Proteomic technology that combines two-dimensional gel electrophoresis (2-DE) and liquid chromatography-tandem mass spectrometry (LC-MS/MS) has high throughput and accuracy, and these technologies are considered as useful tools to comprehensively analyze proteins.

Using these techniques, we found that cofilin-1 displayed differential expression on 2-DE gels between pancreatic cancerous and non-cancerous tissue samples. Western blotting demonstrated that cofilin-1 was significantly up-regulated in cancerous tissues compare to non-cancerous tissues; but opposingly cofilin-2 expression was significantly diminished in cancerous tissues. The findings of the present study incite an interest in cofilin and focus on the relationship between cofilin isoforms and cancer progression.

\section{Materials and methods}

Sample preparation. Twenty-four pairs of pancreatic noncancerous and cancerous tissues were collected from 24 patients diagnosed with PC who underwent surgical resection of the pancreas at the Department of Surgery II, Yamaguchi University Hospital. None of the patients received any preoperative therapy. Written informed consent was obtained from all patients before surgery. The study protocol was approved by the Institutional Review Board for Human Research of the Yamaguchi University School of Medicine.

Tissues were homogenized in lysis buffer (1\% NP-40, $1 \mathrm{mM}$ sodium vanadate, $1 \mathrm{mM}$ PMSF, $10 \mathrm{mM} \mathrm{NaF}, 10 \mathrm{mM}$ EDTA, $50 \mathrm{mM}$ Tris, $165 \mathrm{mM} \mathrm{NaCl}, 10 \mu \mathrm{g} / \mathrm{ml}$ leupeptin and $10 \mu \mathrm{g} / \mathrm{ml}$ aprotinin) on ice (5). After centrifugation $(21,500 \mathrm{x} \mathrm{g}$, $30 \mathrm{~min}, 4^{\circ} \mathrm{C}$ ), the supernatants were used as samples. Ten pairs from the samples were used for 2-DE, and 24 pairs were used for Western blotting. 
Two-dimensional electrophoresis. Isoelectric focusing (IEF) was performed in an IPGphor 3 IEF unit (GE Healthcare, Buckinghamshire, UK) on $11 \mathrm{~cm}$, immobilized linear $\mathrm{pH}$ gradient, pH 3.0-10.0 linear gradient IPG strips (Bio-Rad, Hercules, CA, USA) at $50 \mu \mathrm{A} /$ strip. Samples were mixed with $200 \mu 1$ of rehydration buffer [8 M urea, $2 \%$ CHAPS, $0.01 \%$ bromophenol blue, 1.2\% Destreak reagent (GE Healthcare, Uppsala, Sweden)] and 0.5\% IPG buffer (GE Healthcare) and loaded into the IPGphor strip holder (GE Healthcare). Eighty micrograms of protein was loaded for each 2-DE. The IEF was performed overnight according to the following program: rehydration for $10 \mathrm{~h}(0 \mathrm{~V}) ; 0-500 \mathrm{~V}$ for $4 \mathrm{~h} ; 500-1,000 \mathrm{~V}$ for $1 \mathrm{~h} ; 1,000-8,000 \mathrm{~V}$ for $4 \mathrm{~h} ; 8,000 \mathrm{~V}$ for $20 \mathrm{~min}$; and the final phase of $500 \mathrm{~V}$ from $20,000-30,000 \mathrm{Vh}$ (7).

The IPG strips were equilibrated in equilibration buffer 1 (6 M urea, $0.5 \mathrm{M}$ Tris- $\mathrm{HCl} \mathrm{pH} 8.8,30 \%$ glycerol, $2 \%$ SDS, $2 \%$ 2-ME) for $10 \mathrm{~min}$ and buffer $2(6 \mathrm{M}$ urea, $0.5 \mathrm{M}$ Tris-HCl $\mathrm{pH} 8.8,30 \%$ glycerol, $2 \%$ SDS, $2.5 \%$ iodoacetoamide) for another $10 \mathrm{~min}$. The IPG strips were then transferred onto the gels, run at $200 \mathrm{~V}$; SDS-PAGE was performed on a precastpolyacrylamide gel with a linear concentration gradient of $5-20 \%$ (Bio-Rad) $(8,9)$. Each sample was repeated three times to ensure protein pattern reproducibility.

Fluorescence staining and image analysis. After washing with Milli-Q water 3 times, the SDS-PAGE gels were incubated with $40 \%$ ethanol and $10 \%$ acetic acid for $2.5 \mathrm{~h}$. After fixation, the gel were then stained with a fluorescent gel staining, Flamingo $^{\text {TM }}$ Fluorescent Gel Stain (Bio-Rad) overnight (10). The stained gels were rinsed with Milli-Q water 5 times on a shaker for $5 \mathrm{~min}$. The gels were scanned by using the ProXpress 2-D Proteomic Imaging System (PerkinElmer, Waltham, MA, USA) and then analyzed using Progenesis Samespots software (Nonlinear, Newcastle upon Tyne, UK). Subsequently, the gels were stained with SeePico ${ }^{\mathrm{TM}}$ (Benebiosis, Seoul, Korea) overnight (11). The protein spots showing different intensities were picked up from the gels and analyzed with LC-MS/MS.

Liquid chromatography tandem mass spectrometry analysis. The gel pieces were digested with trypsin and lyophilized overnight with the use of Labconco Lyph-lock 1L Model 77400 (Labconco, Kansas City, MO, USA). Lyophilized samples were dissolved in $15 \mu \mathrm{l}$ of $0.1 \%$ formic acid, and then analyzed using the LC-MS/MS system. Peptide sequencing of the identified protein spot was performed using LC-MS/MS with a Spectrum Mill MS Proteomics Workbench (Agilent Technology, Palo Alto, CA, USA).

Western blotting. Samples were separated by electrophoresis with SDS-PAGE gels and then transfered onto PVDF membranes at $90 \mathrm{~mA}$ for $78 \mathrm{~min}$. The PVDF membranes were blocked overnight with TBS containing $5 \%$ milk at $4^{\circ} \mathrm{C}(12)$. The membranes were incubated with the primary antibody against cofilin-1 (anti-CFL1 mouse monoclonal antibody, 1:1,000; Sigma, St. Louis, MO, USA) or cofilin-2 (anti-CFL2 goat polyclonal antibody, 1:1,000; Santa Cruz Biotechnology, Inc., Santa Cruz, CA) overnight at $4^{\circ} \mathrm{C}$. The membranes were further incubated with the secondary antibody conjugated with horseradish peroxidase $(1: 10,000)$ for $1 \mathrm{~h}$ at room temperature. The membranes were then reacted with a chemiluminescent

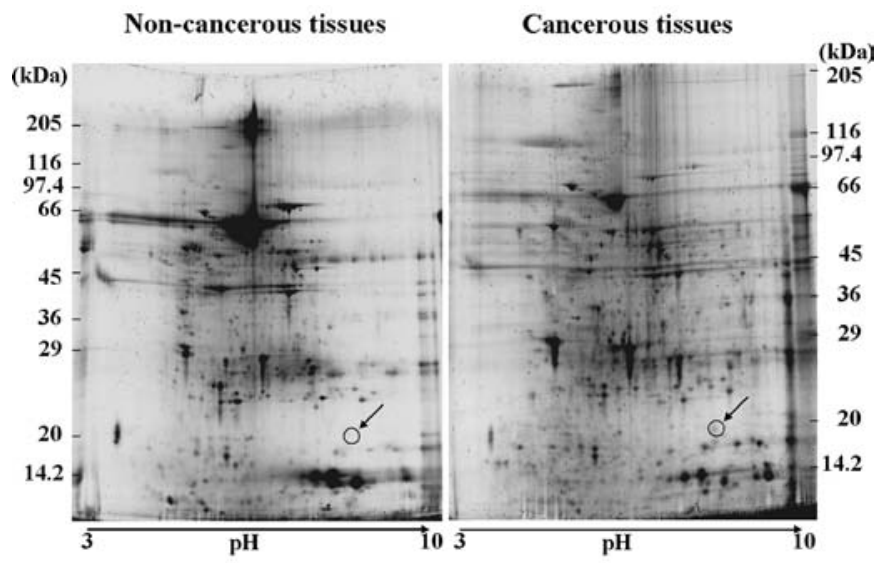

Figure 1. Two-dimensional gel electrophoresis image of pancreatic cancerous and paired non-cancerous tissues stained with Flamingo ${ }^{\mathrm{TM}}$ Gel Staining and scanned using the ProXpress 2-D Proteomic Imaging system. Proteins were separated on $\mathrm{pH}$ 3.0-10.0 linear, immobilized $\mathrm{pH}$ gradient strips and then by $12.5 \%$ SDS-PAGE. The spot (indicated by a circle and an arrow) exhibited a stronger intensity on the gel from the cancerous tissue compared to the noncancerous tissue.

reagent (ImmunoStar Long Detection; Wako, Osaka, Japan) and scanned using the Image Reader LAS-1000 Pro (FujiFilm Corporation, Tokyo, Japan).

\section{Results}

2-DE in the pancreatic non-cancerous and cancerous tissues. 2-DE gels were stained with a fluorescent gel staining and analyzed using Progenesis Samespots software. At least 300 protein spots were matched on each 2-DE gel. A spot of $\sim 20 \mathrm{kDa}$ in mass with an isoelectric point (pI) 8.0 showed stronger intensity in the pancreatic cancerous tissues than in the non-cancerous tissues (Figs. 1 and 2A). The quantification information is summarized in Table I.

Protein identification by $L C-M S / M S$. Cofilin-1 was identified as the up-regulated protein spot on the 2-DE gels. The peptide sequence of cofilin-1 was identified as (K) LGGSAVISLEGKPL(-) by MS and MS/MS spectra of trypsindigested gels. MS/MS data for this protein are summarized in Table I.

Western blot analysis of cofilin isoforms (cofilin-1/2). Cofilin-1 on the 2-DE gels was identified by 2-D gel electrophoresis and Western blotting (Fig. 2B). Twenty-four pairs of cancerous and non-cancerous tissues were analyzed by Western blotting using the anti-cofilin-1 (Fig. 3A) or the anti-cofilin-2 antibody (Fig. 4A). The different intensities of the bands between the cancerous and non-cancerous tissues were quantified by the Student's t-test. The expression of cofilin-1 was increased in the pancreatic cancerous tissues when compared to the noncancerous tissues (22/24; 91.6\%), (Fig. 3A). The intensities of the bands of cofilin-1 in the cancerous and non-cancerous tissue samples were 116.3 and 31.7 ( $\mathrm{p}<0.001$ ), respectively (Fig. 3B). The intensities of the bands of cofilin- 2 in the cancerous and non-cancerous tissues samples were 67.4 and $9.1(\mathrm{p}<0.001)$, respectively (Fig. 4B). The expression of cofilin-2 was 
A

\section{Non-cancerous tissues}

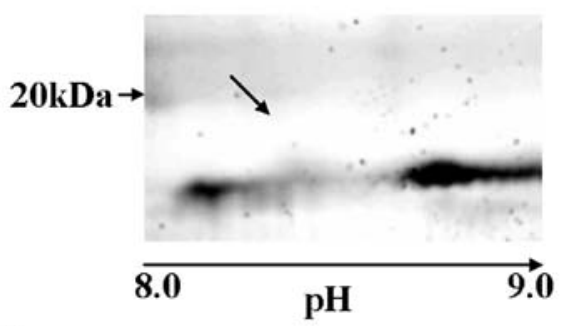

B

\section{Non-cancerous tissues}

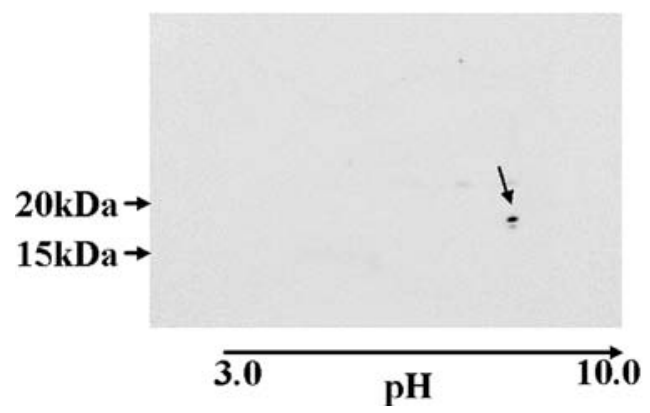

Cancerous tissues

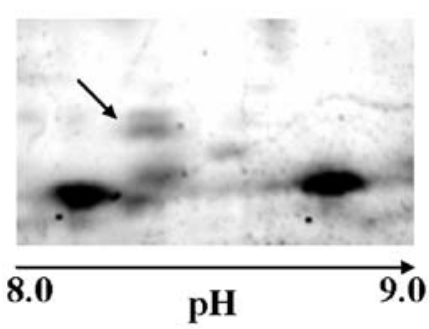

Cancerous tissues

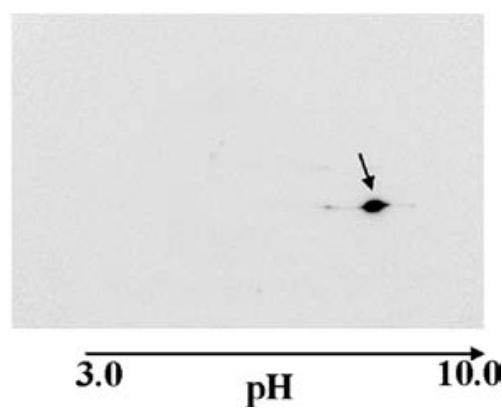

Figure 2. Up-regulated expression of the spot as determined by two-dimensional gel electrophoresis (2-DE) and 2-D Western blotting between cancerous and paired non-cancerous tissues. (A) Differential intensity of the spot between the cancerous tissue compared to the paired non-cancerous tissue as shown on 2-DE gels. (B) Localization of the spot (cofilin-1) was displayed on PDVF membranes by 2-D Western blotting using the anti-cofilin-1 antibody.

Table I. Identification and intensity of the up-regulated protein spot in the cancerous tissues.

\begin{tabular}{lcccccr}
\hline Protein & Accession no. & pI/Mr & Peptide $^{\mathrm{b}}$ & Intensity $^{\mathrm{c}}$ & Frequency $^{\mathrm{a}}$ & $\mathrm{p}^{\mathrm{v} \text {-value }}$ \\
\hline Cofilin-1 & P23528 & $8.22 / 18502.6$ & (K)LGGSAVISLEGKPL(-) & 1.8 & $8 / 10$ & 0.005 \\
\hline
\end{tabular}

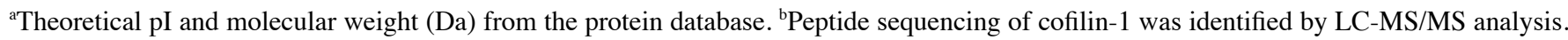

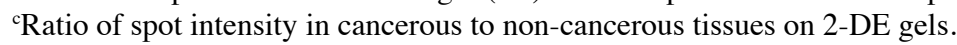
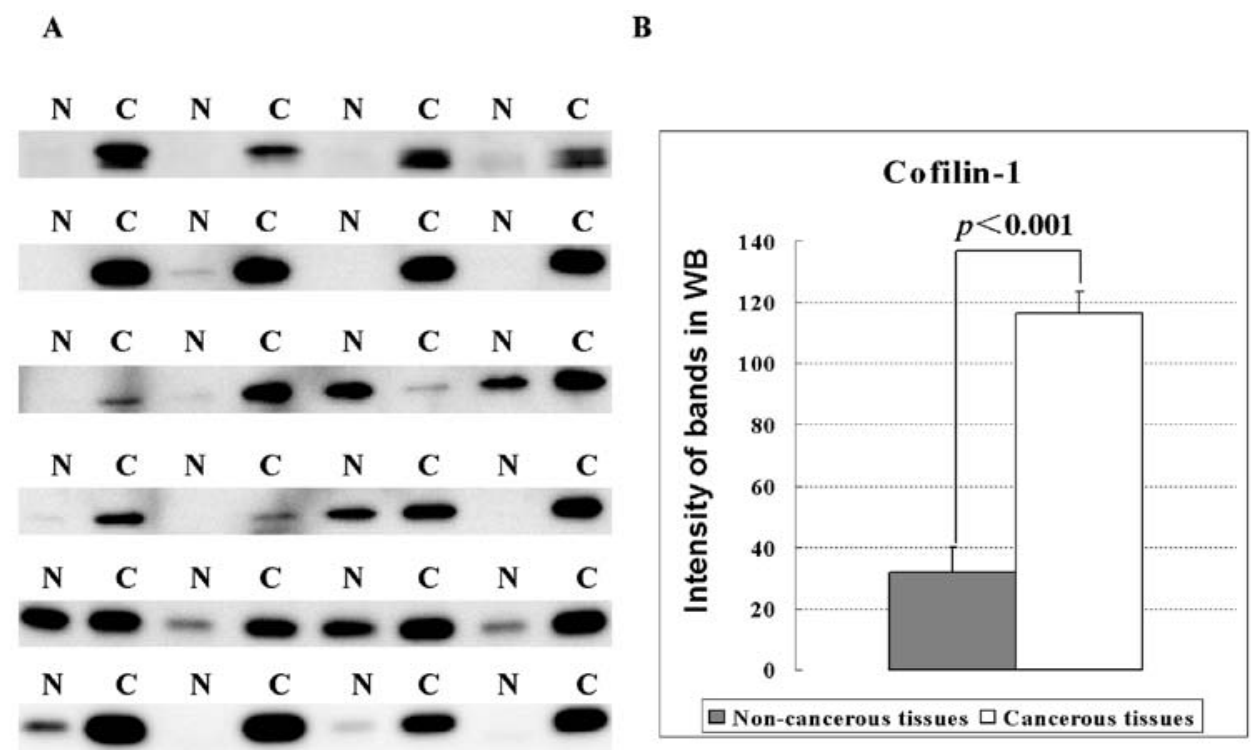

Figure 3. Western blot analysis of cofilin-1 was carried out in pancreatic cancerous and paired non-cancerous tissues. (A) Pancreatic cancerous (C) and paired non-cancerous (N) tissues from 24 PC patients were used, and the anti-cofilin-1 antibody was applied. The expression of cofilin-1 was increased in the pancreatic cancerous tissues compared to that in the paired non-cancerous tissues (91.67\%). (B) Comparison of the intensity of the bands between the cancerous and non-cancerous tissues by the Student's t-test $(n=24, p<0.001)$. The relative standard errors (SE) of cancerous and non-cancerous tissue samples were 8.112 and 6.852 , respectively. 
A

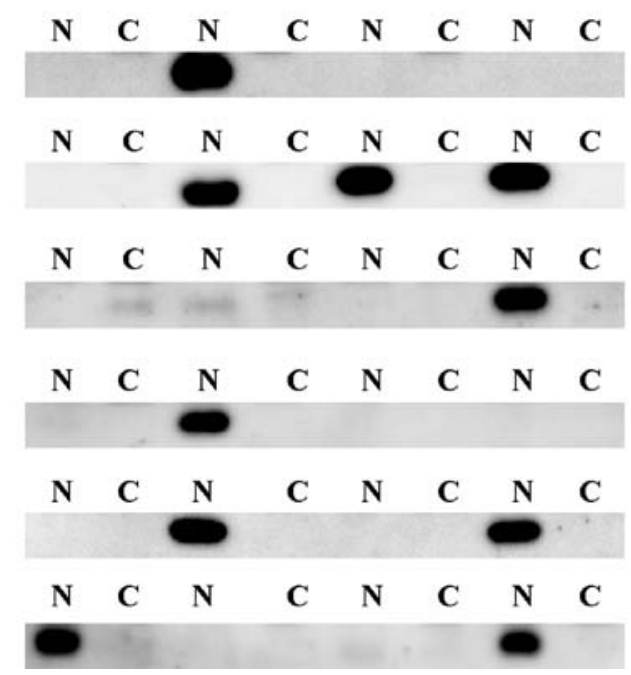

B

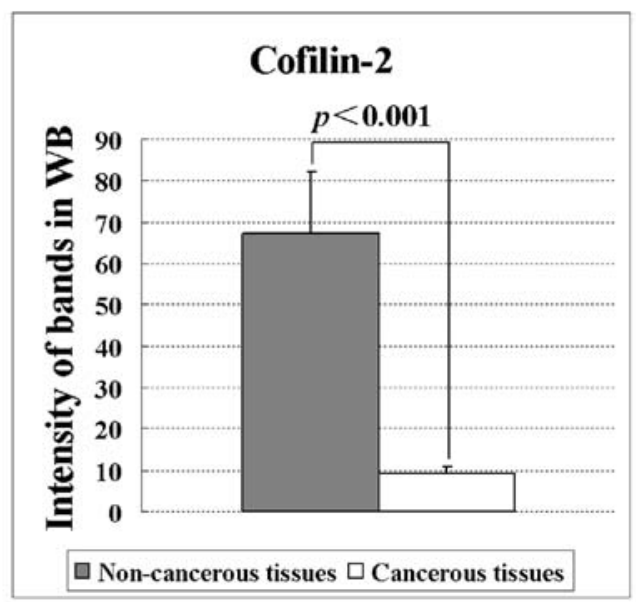

Figure 4. Western blot analysis of cofilin-2 was carried out in pancreatic cancerous and paired non-cancerous tissues. (A) Pancreatic cancerous (C) and paired non-cancerous $(\mathrm{N})$ tissues from 24 PC patients were used, and the anti-cofilin-2 antibody was applied. The expression of cofilin-2 was barely detectable in the pancreatic non-cancerous tissues (58.3\%) and in the cancerous tissues (100\%). (B) Comparison of the intensity of the bands between cancerous and noncancerous tissues by the Student's t-test $(n=24, \mathrm{p}<0.001)$. The relative standard errors (SE) of cancerous and non-cancerous tissue samples were 14.749 and 1.758 , respectively.

detectable in the non-cancerous tissue samples $(10 / 24 ; 41.7 \%)$, but was not in the cancerous tissues (0/24) (Fig. 4A).

\section{Discussion}

LIM domain kinase 1 (LIMK1) and cofilin are important regulators of actin cytoskeleton, and up-regulated actin cytoskeleton enhances tumor cell migration and invasion (13). The LIMK1-mediated cofilin pathway is directly related with mammary tumor invasion and migration (14). Cofilin was found to regulate cell protrusion and motility through the spatial interaction of lamellipodium and lamella (15). Epidermal growth factor (EGF)-induced phosphatidylinositol 4,5-bisphosphate (PIP2) was found to regulate membrane translocation of cofilin in carcinoma cells (16). In the present study, non-muscle cofilin (cofilin-1) was up-regulated in pancreatic cancerous tissues compared with non-cancerous tissues as determined by proteomic profiling. Notably, we demonstrated that expression of the muscle cofilin (cofilin-2), an isoform of cofilin-1, was completely disrupted in pancreatic cancerous tissues by Western blotting.

Cofilin-1, a small ubiquitous protein $(\sim 18.5 \mathrm{kDa})$, regulates actin dynamics through its ability to bind and sever actin filaments during cell migration (17). Cofilin-1 plays roles in cell proliferation, phagocytosis, chemotactic movement and macropinocytosis $(18,19)$. It is generally regarded as an accessory to tumor cell invasion and motility (13). Inhibition of cofilin-1 activity in carcinoma cells reduces cell motility and invasion (20). Cofilin-1 expression was found to be up-regulated in many types of tumor cells, such as invasive mammary tumor cells (21), human glioblastoma cells (22) and the $\mathrm{C} 6$ rat glioblastoma cell line (23). In mammals, actin-depolymerizing factor (ADF)/cofilins are a family of monomeric and filamentous actin binding proteins, consisting of three members, cofilin-1, cofilin-2 and ADF (17,24). Cytoskeletal dynamics and cell motility in mammalian cells require ADF and cofilin-1 activity (20). Thus, cofilin-1 may be involved in motility and invasion of tumor cells in PC.

However, little is known about cofilin-2 in humans. Cofilin-2 accumulates at substrate adhesion sites where cofilin-1 is almost completely excluded (25); a significant increase in cofilin-2 expression was noted during the aggregation stage of cell development under conditions of starvation in Dictyostelium cells. However, cofilin-1 exhibited an opposing and concomitant action (25).

These findings indicate that cofilin- 1 and cofilin-2 may play different roles in the dynamic reorganization of the actin architecture respectively, and cell development may involve the participation of cofilin- 2 under conditions of starvation (25). Cofilin-2 expression in human skeletal muscle and the heart is different from its expression in various tissues in posttranscriptional splicing of mRNA (26). Albeit cofilin-2 gene transcript was detected in the human pancreas; we demonstrated that this protein was barely expressed in pancreatic cancerous tissues (100\%). Notably, not all non-cancerous tissue samples exhibited a high level of cofilin-2 expression; approximately $41.7 \%$ of the non-cancerous tissues showed obviously detectable cofilin-2 expression. The mechanism by which this occurs is not clearly understood. Presumably cofilin-2 exists in non-cancerous tissues only under conditions of starvation at levels required to maintain cell development (25) or survival. Starvation-induced autophagy is a mechanism which promotes cell survival (27). However, it has yet to be revealed whether starvation-induced cell autophagy is related to cofilin-2 expression. However, the expression of cofilin-2 may not be required by well-nourished non-cancerous tissues in PC. Therefore, cofilin-2 may be related to the struggle between the body and tumors under conditions of starvation. Whether cofilin-2 is involved in resistance to starvation by a 
self-regulating protective mechanism in non-cancerous tissue cells during PC progression must be clarified by further study.

The present study suggests that cofilin isoforms (cofilin1/2) play essential roles in the destabilization of the actin cytoskeleton in PC progression involving different pathways. In order to clarify whether cofilin isoforms dysregulated in PC lead to rapid tumor spread, further investigations concerning their expression levels in PC cell lines and the determination of the effects of the cofilin pathways on invasion, proliferation or autophagy of these cells must be carried out. In addition, reports have demonstrated that cofilin expression is related to treatment resistance in PC cell lines $(28,29)$. Therefore, our study indicates that cofilin isoforms may be useful clinical biomarkers or effective targets for controlling PC progression.

\section{Acknowledgements}

This study was supported in part by Grants-in-Aid from the Ministry of Health, Labour and Welfare of Japan (no. H20-Bio-005 to K.N.).

\section{References}

1. Jemal A, Siegel R, Ward E, Hao Y, Xu J and Thun MJ: Cancer statistics, 2009. CA Cancer J Clin 59: 225-249, 2009.

2. Kuramitsu Y, Miyamoto H, Tanaka T, Zhang X, Fujimoto M, Ueda K, Tanaka T, Hamano K and Nakamura K: Proteomic differential display analysis identified upregulated astrocytic phosphoprotein PEA-15 in human malignant pleural mesothelioma cell lines. Proteomics 9: 5078-5089, 2009.

3. Luk JM, Lam CT, Siu AF, Lam BY, Ng IO, Hu MY, Che CM and Fan ST: Proteomic profiling of hepatocellular carcinoma in Chinese cohort reveals heat-shock protein (Hsp27, Hsp70, GRP78) up-regulation and their associated prognostic values. Proteomics 6: 1049-1057, 2006.

4. Roth U, Razawi H, Hommer J, Engelmann K, Schwientek T, Müller S, Baldus SE, Patsos G, Corfield AP, Paraskeva C and Hanisch FG: Differential expression proteomics of human colorectal cancer based on a syngeneic cellular model for the progression of adenoma to carcinoma. Proteomics 10: 194-202, 2010.

5. Kuramitsu Y, Harada T, Takashima M, Yokoyama Y, Hidaka I, Iizuka N, Toda T, Fujimoto M, Zhang X, Sakaida I, Okita K, Oka $\mathrm{M}$ and Nakamura K: Increased expression and phosphorylation of liver glutamine synthetase in well-differentiated hepatocellular carcinoma tissues from patients infected with hepatitis C virus. Electrophoresis 27: 1651-1658, 2006.

6. Wang Y, Kuramitsu Y, Yoshino S, Takashima M, Zhang X, Ueno T, Suzuki N, Oka M and Nakamura K: Screening for serological biomarkers of pancreatic cancer by two-dimensional electrophoresis and liquid chromatography-tandem mass spectrometry. Oncol Rep 26: 287-292, 2011.

7. Tanaka T, Kuramitsu Y, Fujimoto M, Naito S, Oka M and Nakamura K: Downregulation of two isoforms of ubiquitin carboxyl-terminal hydrolase isozyme L1 correlates with high metastatic potentials of human SN12C renal cell carcinoma cell clones. Electrophoresis 29: 2651-2659, 2008.

8. Kuramitsu Y, Hayashi E, Okada F, Tanaka T,Zhang X, Ueyama Y and Nakamura K: Proteomic analysis for nuclear proteins related to tumour malignant progression: a comparative proteomic study between malignant progressive cells and regressive cells. Anticancer Res 30: 2093-2099, 2010.

9. Kuramitsu Y, Baron B, Yoshino S, Zhang X, Tanaka T, Yashiro M, Hirakawa K, Oka M and Nakamura K: Proteomic differential display analysis shows up-regulation of 14-3-3 protein sigma in human scirrhous-type gastric carcinoma cells. Anticancer Res 30: 4459-4465, 2010.

10. Kuramitsu Y, Taba K, Ryozawa S, Yoshida K, Tanaka T, Zhang X, Maehara S, Maehara Y, Sakaida I and Nakamura K: Identification of up- and down-regulated proteins in gemcitabine-resistant pancreatic cancer cells using two-dimensional gel electrophoresis and mass spectrometry. Anticancer Res 30: 3367-3372, 2010.
11. Kuramitsu Y, Hayashi E, Okada F, Zhang X, Tanaka T, Ueyama Y and Nakamura K: Staining with highly sensitive Coomassie brilliant blue SeePico ${ }^{\mathrm{TM}}$ stain after Flamingo ${ }^{\mathrm{TM}}$ fluorescent gel stain is useful for cancer proteomic analysis by means of twodimensional gel electrophoresis. Anticancer Res 30: 4001-4005, 2010.

12. Mori-Iwamoto S, Kuramitsu Y, Ryozawa S, Mikuria K, Fujimoto M, Maehara S, Maehara Y, Okita K, Nakamura K and Sakaida I: Proteomics finding heat shock protein 27 as a biomarker for resistance of pancreatic cancer cells to gemcitabine. Int J Oncol 31: 1345-1350, 2007.

13. Yamaguchi $\mathrm{H}$ and Condeelis $\mathrm{J}$ : Regulation of the actin cytoskeleton in cancer cell migration and invasion. Biochim Biophys Acta 1773: 642-652, 2007.

14. Wang W, Mouneimne G, Sidani M, Wyckoff J, Chen X, Makris A, Goswami S, Bresnick AR and Condeelis JS: The activity status of cofilin is directly related to invasion, intravasation, and metastasis of mammary tumors. J Cell Biol 173: 395-404, 2006.

15. Delorme V, Machacek M, DerMardirossian C, Anderson KL, Wittmann T, Hanein D, Waterman-Storer C, Danuser G and Bokoch GM: Cofilin activity downstream of Pak1 regulates cell protrusion efficiency by organizing lamellipodium and lamella actin networks. Dev Cell 13: 646-662, 2007.

16. van Rheenen J, Song X, van Roosmalen W, Cammer M, Chen X, Desmarais V, Yip SC, Backer JM, Eddy RJ and Condeelis JS: EGF-induced PIP2 hydrolysis releases and activates cofilin locally in carcinoma cells. J Cell Biol 179: 1247-1259, 2007.

17. Bamburg JR: Proteins of the ADF/cofilin family: essential regulators of actin dynamics. Annu Rev Cell Dev Biol 15: 185-230, 1999.

18. Aizawa H, Sutoh K, Tsubuki S, Kawashima S, Ishii A and Yahara I: Identification, characterization, and intracellular distribution of cofilin in Dictyostelium discoideum. J Biol Chem 270: 10923-10932, 1995.

19. Aizawa H, Sutoh K and Yahara I: Overexpression of cofilin stimulates bundling of actin filaments, membrane ruffling, and cell movement in Dictyostelium. J Cell Biol 132: 335-344, 1996.

20. Hotulainen P, Paunola E, Vartiainen MK and Lappalainen P: Actin-depolymerizing factor and cofilin-1 play overlapping roles in promoting rapid $\mathrm{F}$-actin depolymerization in mammalian nonmuscle cells. Mol Biol Cell 16: 649-664, 2005.

21. Wang W, Goswami S, Lapidus K, Wells AL, Wyckoff JB, Sahai E, Singer RH, Segall JE and Condeelis JS: Identification and testing of a gene expression signature of invasive carcinoma cells within primary mammary tumors. Cancer Res 64: 8585-8594, 2004.

22. Yap CT, Simpson TI, Pratt T, Price DJ and Maciver SK: The motility of glioblastoma tumour cells is modulated by intracellular cofilin expression in a concentration-dependent manner. Cell Motil Cytoskeleton 60: 153-165, 2005.

23. Gunnersen JM, Spirkoska V, Smith PE, Danks RA and Tan SS: Growth and migration markers of rat C6 glioma cells identified by serial analysis of gene expression. Glia 32: 146-154, 2000.

24. Vartiainen MK, Mustonen T, Mattila PK, Ojala PJ, Thesleff I, Partanen $\mathbf{J}$ and Lappalainen P: The three mouse actin-depolymerizing factor/cofilins evolved to fulfill cell-type-specific requirements for actin dynamics. Mol Biol Cell 13: 183-194, 2002.

25. Aizawa H, Kishi Y, Iida K, Sameshima M and Yahara I: Cofilin-2, a novel type of cofilin, is expressed specifically at aggregation stage of Dictyostelium discoideum development. Genes Cells 6: 913-921, 2001.

26. Thirion C, Stucka R, Mendel B, Gruhler A, Jaksch M, Nowak KJ, Binz N, Laing NG and Lochmüller H: Characterization of human muscle type cofilin (CFL2) in normal and regenerating muscle. Eur J Biochem 268: 3473-3482, 2001.

27. Codogno P: Autophagy in cell survival and death. J Soc Biol 199: 233-241, 2005.

28. Sinha P, Hütter G, Köttgen E, Dietel M, Schadendorf D and Lage $\mathrm{H}$ : Increased expression of epidermal fatty acid binding protein, cofilin, and 14-3-3-sigma (stratifin) detected by two-dimensional gel electrophoresis, mass spectrometry and microsequencing of drug-resistant human adenocarcinoma of the pancreas. Electrophoresis 20: 2952-2960, 1999.

29. Cecconi D, Astner H, Donadelli M, Palmieri M, Missiaglia E, Hamdan M, Scarpa A and Righetti PG: Proteomic analysis of pancreatic ductal carcinoma cells treated with 5-aza-2'-deoxycytidine. Electrophoresis 24: 4291-4303, 2003. 